\title{
VirHunter: a deep learning-based method for detection of novel viruses in plant sequencing data
}

Sukhorukov G. ${ }^{1,2 *}$, Nikolski M. ${ }^{1,2}$

${ }^{1}$ University of Bordeaux, CNRS, IBGC, UMR 5095, Bordeaux, France

${ }^{2}$ University of Bordeaux, Bordeaux Bioinformatics Center, Bordeaux, France

*email: grigorii.sukhorukov@u-bordeaux.fr

Plant viruses are a major plant pathogen; plant infections by viruses result in more than $\$ 30$ billion losses a year. One of the best ways to fight against plant infections caused by viruses is the surveillance and early detection of viral presence in plant populations. For this task high-throughput sequencing (HTS) is often used as an excellent tool to investigate the viral presence in large plant samples. However HTS data comprises a mixture of sequences containing not only viral sequences but also contaminating sequences coming from the host and bacteria. The downstream bioinformatics analysis of these data can be difficult and time consuming. Moreover, computational tools often miss certain viruses present in the sample, especially the previously uncharacterised viral species. To help solve this task we have developed an artificial neural network approach that classifies sequences as belonging to viral, bacterial or plant host origin and is implemented in the Keras framework. The developed approach uses the one-hot encoding for both sequences and their reverse complement and relies on convolutional layers to learn k-mers distinguishing viral sequences. The network was trained on a dataset composed of three balanced classes corresponding to plant viruses, plants and bacteria. To be performant our VirHunter method has to be trained for the analysis of a specific plant's virome, as the training dataset is generated using the host information. We test our tool in different settings assessing its ability to detect novel viruses. Finally, we compare the performance of our tool to that of DeepVirFinder, a previously developed deep learning-based method for virus detection. 